\title{
Validation of the Publication of New Names and New Combinations Previously Effectively Published Outside the IJSB
}

\author{
List No. $61 \dagger$
}

The purpose of this announcement is to effect the valid publication of the following new names and new combinations under the procedure described previously [Int. J. Syst. Bacteriol. 27(3):iv, 1977]. Authors and other individuals wishing to have new names and/or combinations included in future lists should send the pertinent reprint or a photocopy thereof to the IJSB (c/o ASM) for confirmation that all of the other requirements for valid publication have been met. It should be noted that the date of valid publication of these new names and combinations is the date of publication of this list, not the date of the original publication of the names and combinations. The authors of the new names and combinations are as given below, and these authors' names will be included in the author index of the present issue and in the volume author index in this issue of the IJSB.

Inclusion of a name on these lists validates the name and thereby makes it available in bacteriological nomenclature. The inclusion of a name on this list is not to be construed as taxonomic acceptance of the taxon to which the name is applied. Indeed, some of these names may, in time, be shown to be synonyms, or the organisms may be transferred to another genus, thus necessitating the creation of a new combination.

\begin{tabular}{|c|c|c|c|c|}
\hline Name & Proposed as: & Author(s) (reference) & Priority $^{a}$ & Nomenclatural type ${ }^{b}$ \\
\hline Alteromonas elykovii & New species & Ivanova et al. (12) & 1 & $\begin{array}{l}\text { Strain 40MC }(=\mathrm{KMM} 162=\mathrm{VKPM} \\
\text { B3905) }\end{array}$ \\
\hline Desulfocapsa & New genus & Janssen et al. (14) & 2 & Desulfocapsa thiozymogenes \\
\hline Desulfocapsa thiozymogenes & New species & Janssen et al. (14) & 2 & Strain Bra2 (= DSM 7269) \\
\hline Desulfuromonas acetexigens & New species & Finster et al. (4) & 5 & Strain 2873 (= DSM $\left.1397^{\circ}\right)$ \\
\hline Ferroglobus & New genus & Hafenbradl et al. (10) & 3 & Ferroglobus placidus \\
\hline Ferroglobus placidus & New species & Hafenbradl et al. (10) & 3 & Strain AEDII12DO (= DSM 10642) \\
\hline Flexibacter japonensis & New species & Fujita et al. (8) & 1 & Strain 758 (= JCM 9735) \\
\hline Halomonas pantelleriense & New species & Romano et al. (17) & 11 & Strain AAP (= DSM 9661) \\
\hline Helicobacter bilis & New species & Fox et al. (6) & 11 & Strain Hb1 $(=$ ATCC 51630) \\
\hline Helicobacter cholecystus & New species & Franklin et al. (7) & 9 & Strain Hkb-1 \\
\hline Lactobacillus lindneri & New species, revived name & Back et al. (1) & 8 & Strain KPA (= DSM 20690) \\
\hline Leptothrix discophora & New species, revived name & Spring et al. (18) & 8 & Strain SS-1 (= LMG 8141) \\
\hline Leptothrix mobilis & New species & Spring et al. (18) & 8 & Strain Feox-1 (= LMG 17066) \\
\hline Methanobrevibacter curvatus & New species & $\begin{array}{l}\text { Leadbetter and Breznak } \\
\text { (16) }\end{array}$ & 1 & Strain RFM-2 (= DSM 11111) \\
\hline Methanobrevibacter cuticularis & New species & $\begin{array}{l}\text { Leadbetter and Breznak } \\
\text { (16) }\end{array}$ & 1 & Strain RFM-1 (= DSM 11139) \\
\hline Mycobacterium triplex & New species & Floyd et al. (5) & 6 & Strain $90-1019\left(=\right.$ ATCC $\left.700071^{d}\right)$ \\
\hline $\begin{array}{l}\text { Pseudomonas avellanae (formerly } \\
\text { Pseudomonas syringae pv. } \\
\text { avellanae) }\end{array}$ & New species & Janse et al. (13) & 8 & Strain F11 (= NCPPB 3487) \\
\hline Pseudomonas rhodesiae & New species & Coroler et al. (3) & 8 & CIP 104664 \\
\hline Rhodobacter azotoformans & New species & Hiraishi et al. (11) & 6 & Strain KA25 (= JCM 9340) \\
\hline Ruminococcus hydrogenotrophicus & New species & Bernalier et al. (2) & 7 & Strain S5a33 (= DSM 10507) \\
\hline Selenomonas acidaminovorans & New species & Guangsheng et al. (9) & 4 & Strain Su883 (= DSM 6589) \\
\hline Sphingomonas herbicidovorans & New species & Zipper et al. (19) & 10 & Strain MH (= DSM 11019) \\
\hline Thermococcus alcaliphilus & New species & Keller et al. (15) & 3 & Strain AEDII12 ( = DSM 10322) \\
\hline
\end{tabular}

$\dagger$ Lists 1 through 60 were published in the Int. J. Syst. Bacteriol. 27:306, 1977; 29:79, 436, 1979; 30:601, 676, 1980; 31:215, 382, 1981; 32:266, 384, 1982; 33:438, 672, 896,$1983 ; \mathbf{3 4}: 91$, 270, 355, 503, 1984; 35:223, 375, 535, 1985; 36:354, 489, 573, 1986; 37:179, 1987; 38:136, 220, 328, 449, 1988; 39:93, 205, 371, 495, 1989; 40:105, 212, $320,470,1990 ; 41: 178,331,456,580,1991 ; 42: 191,327,511,656,1992 ; 43: 188,398,624,864,1993 ; 44: 182,370,595,852,1994 ; 45: 197,418,619,879,1995 ; 46: 362,625$, $836,1189,1996 ;$ and 47:242, 1997.

${ }^{a}$ Priority number assigned according to the date the documentation and request for validation are received.

${ }^{b}$ Abbreviations: ATCC, American Type Culture Collection, Rockville, Md.; CIP, Collection of the Institute Pasteur, Paris, France; DSM, DSMZ-Deutsche Sammlung von Mikroorganismen und Zellkulturen GmbH, Braunschweig, Germany; JCM, Japan Collection of Microorganisms, Riken, Saitama, Japan; KMM Collection of Marine Microorganisms, Pacific Institute of Bioorganic Chemistry, Far-Eastern Branch of the Russian Academy of Sciences, Vladivostok, Russia; LMG, LMG Culture Collection, Universiteit Gent, Ghent, Belgium; NCPPB, National Collection of Plant Pathogenic Bacteria, Harpenden, England; VKPM, Russian Collection of Industrial Microorganisms, Moscow, Russia.

${ }^{c}$ The wrong accession number, DSM 1379, given in the effective publication has been corrected to DSM 1397.

${ }^{d}$ The wrong accession number, ATCC 70071, given in the effective publication has been corrected to ATCC 700071 .

\section{REFERENCES}

1. Back, W., I. Bohak, M. Ehrmann, W. Ludwig, and K. H. Schleifer. 1996 Revival of the species Lactobacillus lindneri and the design of a species specific oligonucleotide probe. Syst. Appl. Microbiol. 19:322-325.

2. Bernalier, A., A. Willems, M. Leclerc, V. Rochet, and M. D. Collins. 1996. Ruminococcus hydrogenotrophicus sp. nov., a new $\mathrm{H}_{2} / \mathrm{CO}_{2}$-utilizing acetogenic bacterium isolated from human feces. Arch. Microbiol. 166:176-183.
3. Coroler, L., M. Elomari, B. Hoste, M. Gillis, D. Izard, and H. Leclerc. 1996. Pseudomonas rhodesiae sp. nov., a new species isolated from natural mineral waters. Syst. Appl. Microbiol. 19:600-607.

4. Finster, K., F. Bak, and N. Pfennig. 1994. Desulfuromonas acetexigens sp. nov., a dissimilatory sulfur-reducing eubacterium from anoxic freshwater sediments. Arch. Microbiol. 161:328-332.

5. Floyd, M. M., L. S. Guthertz, V. A. Silcox, P. S. Duffey, Y. Jang, E. P. 
Desmond, J. T. Crawford, and W. R. Butler. 1996. Characterization of an SAV organism and proposal of Mycobacterium triplex sp. nov. J. Clin. Microbiol. 34:2963-2967.

6. Fox, J. G., L. L. Yan, F. E. Dewhirst, B. J. Paster, B. Shames, J. C. Murphy, A. Hayward, J. C. Belcher, and E. N. Mendes. 1995. Helicobacter bilis sp. nov, a novel Helicobacter species isolated from bile, livers, and intestines of aged, inbred mice. J. Clin. Microbiol. 33:445-454.

7. Franklin, C. L., C. S. Beckwith, R. S. Livingston, L. K. Riley, S. V. Gibson, C. L. Besch-Williford, and R. R. Hook, Jr. 1996. Isolation of a novel Helcobacter species, Helicobacter cholecystus sp. nov., from the gallbladders of Syrian hamsters with cholangiofibrosis and centrilobular pancreatitis. J. Clin. Microbiol. 34:2952-2958.

8. Fujita, T., M. Okamoto, Y. Kosako, and M. Okuhara. 1996. Flexibacter japonensis sp. nov., a new species that produces a novel inhibitor of human leukocyte elastase isolated from soil. Curr. Microbiol. 33:89-93.

9. Guangsheng C. C. M. Plugge, W. Roelofsen, F. P. Houwen, and A. J. M. Stams. 1992. Selenomonas acidaminovorans sp. nov., a versatile thermophilic proton-reducing anaerobe able to grow by decarboxylation of succinate to propionate. Arch. Microbiol. 157:169-175.

10. Hafenbradl, D., M. Keller, R. Dirmeier, R. Rachel, P. Roßnagel, S. Burggraf, and K. O. Stetter. 1996. Ferroglobus placidus gen. nov., sp. nov., a novel hyperthermophilic archaeum that oxidizes $\mathrm{Fe}^{2+}$ at neutral $\mathrm{pH}$ under anoxic hyperthermophilic archaeum that oxidizes

11. Hiraishi, A., K. Muramatsu, and Y. Ueda. 1996. Molecular genetic analyses of Rhodobacter azotoformans sp. nov. and related species of phototrophic bacteria. Syst. Appl. Microbiol. 19:168-177.

12. Ivanova, E. P., V. V. Mikhailov, E. A. Kiprianova, G. F. Levanova, A. D. Garagulya, G. M. Frolova, and V. I. Svetashev. 1996. Alteromonas elyakovii sp. nov.-a new bacterium isolated from marine mollusks. Biologiya Mory
(Russian Journal of Marine Biology, Vladivostok) 22:213-237.

13. Janse, J. D., P. Rossi, L. Angelucci, M. Scortichini, J. H. J. Derks, A. D. L Akkermans, R. de Vrijer, and P. G. Psallidas. 1996. Reclassification of Pseudomonas syringae pv. avellanae as Pseudomonas avellanae (spec. nov.) the bacterium causing canker of hazelnut (Corylus avellana L.). Syst. Appl. Microbiol. 19:589-595.

14. Janssen, P. H., A. Schuhmann, F. Bak, and W. Liesack. 1996. Dispropor tionation of inorganic sulfur compounds by the sulfate-reducing bacterium Desulfocapsa thiozymogenes gen. nov., sp. nov. Arch. Microbiol. 166:184-192.

15. Keller, M. F.-J. Braun, R. Dirmeier, D. Hafenbradl, S. Burggraf, and K. O. Stetter. 1995. Thermococcus alcaliphilus sp. nov., a new hyperthermophilic Stetter. 1995. Thermococcus alcaliphilus sp. nov., a new hyperthermophilic
archaeum growing on polysulfide at alkaline pH. Arch. Microbiol. 164:390395 .

16. Leadbetter, J. R., and J. A. Breznak. 1996. Physiological ecology of Methanobrevibacter cuticularis sp. nov. and Methanobrevibacter curvatus sp. nov., isolated from the hindgut of the termite Reticulitermes flavipes. Appl. Environ. Microbiol. 62:3620-3631.

17. Romano, I., B. Nicolaus, L. Lama, M. C. Manca, and A. Gambacorta. 1996 Characterization of a haloalkalophilic strictly aerobic bacterium, isolated from Pantelleria island. Syst. Appl. Microbiol. 19:326-333.

18. Spring, S., P. Kämpfer, W. Ludwig, and K. H. Schleifer. 1996. Polyphasic characterization of the genus Leptothrix: new descriptions of Leptothrix mobilis sp. nov. and Leptothrix discophora sp. nov. nom. rev. and emended description of Leptothrix cholodnii emend. Syst. Appl. Microbiol. 19:634-643.

19. Zipper, C., K. Nickel, W. Angst, and H.-P. E. Kohler. 1996. Complete microbial degradation of both enantiomers of the chiral herbicide mecoprop $[(R S)$-2-(4-chloro-2-methylphenoxy)propionic acid] in an enantioselective manner by Sphingomonas herbicidovorans sp. nov. Appl. Environ. Microbiol. 62: $4318-4322$ 